\title{
Interaction of Refractory Dibenzothiophenes and Polymerizable Structures
}

\author{
Jose L. Rivera, ${ }^{1}$ Pedro Navarro-Santos, ${ }^{2}$ Roberto Guerra-Gonzalez, ${ }^{1}$ and Enrique Lima ${ }^{3}$ \\ ${ }^{1}$ Facultad de Ingeniería Química, Universidad Michoacana de San Nicolás de Hidalgo, 58000 Morelia, MICH, Mexico \\ ${ }^{2}$ Facultad de Ingeniería en Tecnología de la Madera, Universidad Michoacana de San Nicolás de Hidalgo, \\ 58000 Morelia, MICH, Mexico \\ ${ }^{3}$ Instituto de Investigaciones en Materiales, UNAM, Ciudad Universitaria, 04510 México, DF, Mexico \\ Correspondence should be addressed to Jose L. Rivera; jlrivera@umich.mx
}

Received 4 November 2013; Accepted 4 March 2014; Published 12 June 2014

Academic Editor: Haojun Liang

Copyright (C) 2014 Jose L. Rivera et al. This is an open access article distributed under the Creative Commons Attribution License, which permits unrestricted use, distribution, and reproduction in any medium, provided the original work is properly cited.

\begin{abstract}
We carried out first principles calculations to show that polymerizable structures containing hydroxyl (alcoholic chain) and amino groups are suitable to form stable complexes with dibenzothiophene (DBT) and its alkyl derivates. These sulfur pollutants are very difficult to eliminate through traditional catalytic processes. Spontaneous and exothermic interactions at $0 \mathrm{~K}$ primarily occur through the formation of stable complexes of organosulfur molecules with monomeric structures by hydrogen bonds. The bonds are formed between the sulfur atom and the hydrogen of the hydroxyl group; additional hydrogen bonds are formed between the hydrogen atoms of the organosulfur molecule and the nitrogen atoms of the monomers. We vary the number of methylene groups in the alcoholic chain containing the hydroxyl group of the monomer and find that the monomeric structure with four methylene groups has the best selectivity towards the interaction with the methyl derivates with reference to the interaction with DBT. Even this study does not consider solvent and competitive adsorption effects; our results show that monomeric structures containing amino and hydroxyl groups can be used to develop adsorbents to eliminate organosulfur pollutants from oil and its derivates.
\end{abstract}

\section{Introduction}

The content of sulfur in liquid fuels has been continuously reduced in the past 3 decades through tighter government restrictions in liquid fuel specifications [1-3]. However, due to the continuous increase in the amount of fuels consumed, the total amount of sulfur released to the environment has not decreased significantly. In the United States, recent government restrictions set a limit for a maximum sulfur content of 15 and 30 parts per million on weights of gasoline and diesel fuel, respectively [4-7]. To continue reducing the sulfur content of fuels, new technologies for desulfurization need to be developed. Sulfur exists naturally in oil in the form of hydrogen sulfide and organosulfur compounds, for example, thiophenes, benzothiophenes, dibenzothiophenes (DBTs), and their alkyl derivates [8]. Hydrodesulfurization (HDS) and dehydrodesulfurization (DDS) processes are commonly employed to separate the sulfur from oil in the form of hydrogen sulfide $[9,10]$. HDS and DDS processes use catalysts that absorb the organosulfur compounds to the catalytic surface through a sulfur atom in the first step. In the second step, the sulfur atoms react with hydrogen and finally the hydrogen sulfide and the corresponding hydrogenated organic compound desorb. These processes transform most of the organosulfur compounds, but some alkyl derivates of DBT are very difficult to absorb and transform [11-13].

Organosulfur compounds formed of sulfur atoms attached to alkane chains are easily processed by HDS and DDS because the sulfur atom is highly accessible to the catalytic surface. Small cyclic compounds containing sulfur, that is, thiophenes and benzothiophenes and their alkyl derivates, are also easily absorbed by the catalytic surfaces and transformed later. Starting from thiophene, which is the smallest cyclic organosulfur compound, the absorption and transformation capacities for those compounds decrease as the number of benzyl and alkyl groups attached to the basic thiophene 
structure increases [4]. The lowest absorption capacity corresponds to 4,6-dimethyldibenzothiophene (4,6-DMDBT) [14]. Among all the possible methyl derivates of DBT present in oil, 4,6-DMDBT and 4-methyldibenzothiophene (4MDBT) have proven to be the most difficult to eliminate from oil and its refined liquid products [4]. They are the main constituents of the remaining sulfur in liquid fuels. Any improvement in desulfurization using catalytic desulfurization technology, which is not very effective on 4-DMBT and 4,6DMDBT compounds, requires higher hydrogen consumption, additional infrastructure in the processing refineries, and the improvement or development of new catalysts [5-7].

New experimental and theoretical approaches to eliminate DBT and its alkyl derivates (other than catalytic approaches) have been developed in the past decade that include the transformation of organosulfur compounds through digestion by microorganisms [15-17], the sonochemical degradation [18-20], the formation of charge transfer complexes [21-23], and the adsorption on modified surfaces containing metallic cations, that is, zeolites [24-26] and silica [27]. Additionally aerogels [28-30], hydrogels [31, 32], and activated graphite with functionalized surfaces containing oxygen-based functional groups and metals [33-35] have been used. These new approaches have been partially successful at eliminating DBT, but some of them are too expensive to apply at a commercial scale.

In this work, we study the interaction of the complexes of problematic organosulfur compounds on several polymerizable structures using density functional theory (DFT) calculations [36-38]. The first monomeric structure we study is formed by a polymerizable segment, which contains one hydroxyl and two primary amino groups. We find that the DBT and its alkyl derivates form stable complexes with these monomeric structures. Complexes are formed via hydrogen bonds at several sites in the monomer and organosulfur compounds; in the monomer, the oxygen, some nitrogen and hydrogen atoms are involved in the formation of hydrogen bonds, while in the organosulfur molecules the sulfur and some hydrogen atoms participate in the hydrogen bonds. The hydroxyl group of the monomeric structure is attached to the main chain through a methylene group and it is not very accessible to the sulfur atom, especially for 4,6-DMDBT. To make the hydroxyl group less and more accessible to the sulfur atom, we modify the original structure and propose another new five monomeric structures. The optimal methylene chain length of monomer that maximizes the complexation energy of organosulfur compounds is discussed in terms of the energetic stability and the selectivity; even this study is not considering the solvent and competitive adsorption effects. In order to explore new avenues of pollutant separation research, it is necessary to gain insight about the intrinsic activity of organosulfur compounds in the presence of hydroxyl or amino groups, which are also present in many adsorbent materials.

This paper is organized as follows: the employed methodology is described in Section 2 and results of geometry optimizations and electronic structure calculations of DBT, 4-MDBT, and 4,6-DMDBT are presented in Section 3. The electronic structure of the first studied monomer is shown in Section 4. Section 5 presented the calculation of the complexation energies of the organosulfur compounds on an initial monomeric structure and compared it with the complexation energies of derivates of the initial structure to elucidate the most appropriate monomeric structure that could be used in the adsorption of organosulfur compounds. Finally, conclusions of this work are shown in Section 6.

\section{Methodology}

We perform geometry optimizations of the organosulfur molecules at the B3LYP/6-311+G(d,p) level of theory using the GAUSSIAN software [39]. The well-known B3LYP [40, 41] hybrid functional consists of Becke exchange functional, the Lee-Yang-Parr correlation functional, and a HF exchange term. This level of theory is competitive in terms of accuracy when it is combined with an adequate basis set as the one used in this work. McAllister [42] found that DFT, using nonlocal and gradient corrected functional, performed very similar to other correlated methods, such as calculations at the MP2 level of theory. In addition, calculations employing this functional have been proved in selected reactions involving sulfur atoms, such as $\mathrm{S}-\mathrm{H}$ bond dissociation enthalpies compared with the experimental gas-phase enthalpy [43], the molar enthalpy of formation [44], and the proton affinities [45]. In particular, Singh et al. [46] have reported the ground state geometry of thiophene and its hydrogen bonded complexes with dimethyl sulfoxide and methanol in gas phase, finding that B3LYP yields better estimate of vibrational wavenumbers of complexes, dipole-dipole interaction of the hydrogen bonded complexes having insignificant differences of energy (up to $0.014 \mathrm{kcal} / \mathrm{mol}$ ) compared with those calculated using the second order Møller-Plesset method (MP2). Finally, the B3LYP functional has been widely used to investigate the potential energy maps of various systems containing carbon, hydrogen, oxygen, and sulfur atoms [47-54].

\section{Electronic Structure of DBT, 4-MDBT, and 4,6-DMDBT}

To identify potentially viable adsorbent materials, we first need information about the organosulfur compounds: electronic structure and energetics. Reliable information about their electronic structure (geometry, electronic density, electrostatic potential, dipole moments, etc.) and energetics of adsorption processes of molecules at surfaces and macromolecules is commonly obtained by $a b$ initio and DFT calculations.

We have compared the optimized geometries of the organosulfur molecules with respect to a more sophisticated methodology as the second order Møller-Plesset (MP2) energy calculation, finding differences in total energy up to $\sim 0.3 \%$ (found in the geometry of the 4,6 -DMDBT); due to the size of the studied systems, MP2 requires a computational effort which is nonpractical [36-38, 55]. The optimized geometries were characterized as true minima by harmonic frequency analysis. More specifically, using this approach, the optimized geometry of DBT exhibits small deviations from 


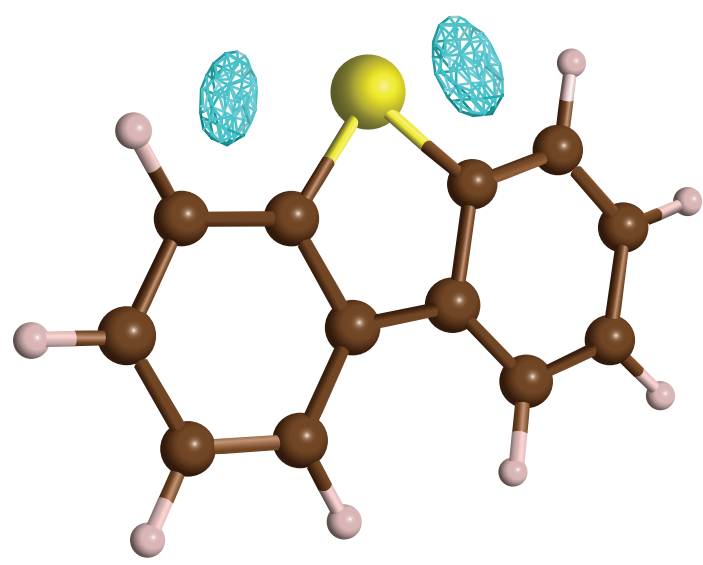

(a)

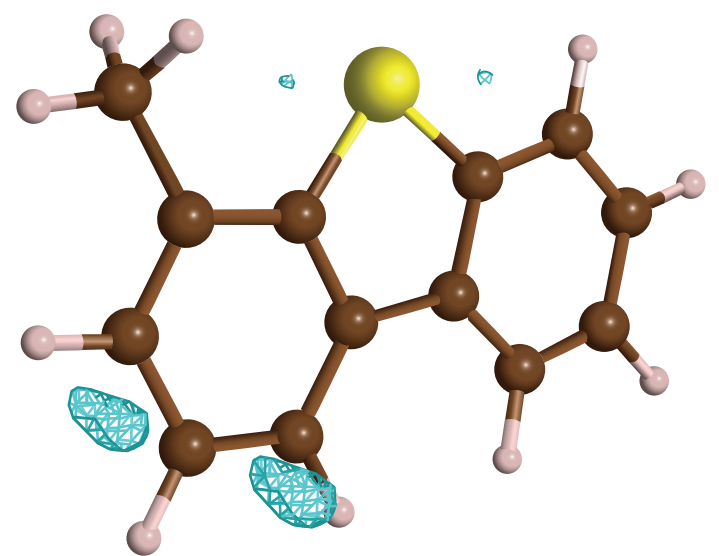

(b)

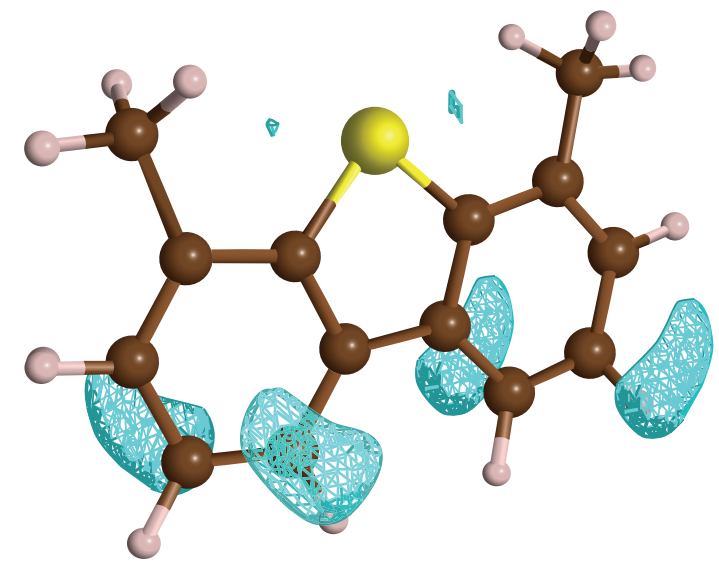

(c)

FIGURE 1: Molecular structures including isosurfaces of the electronic density at constant $V(-55.79 \mathrm{~kJ} / \mathrm{mol})$ for (a) DBT, and (-60.39 kJ/mol) for (b) 4-MDBT, and (c) 4,6-DMDBT. Brown spheres in the molecular structure represent carbon atoms, while white and yellow spheres represent hydrogen and sulfur atoms, respectively.

previously reported geometries using theoretical [56] and experimental approaches [57]; for example, bond distances exhibit differences with the experimental values in the range of 0.001 and $0.020 \AA$, while valence (bond) angles exhibit differences up to $1^{\circ}$. Given the rather minute deviations in geometry, we expect that our results should be quantitatively informative. The molecule is completely flat in agreement with the experimental values. No experimental geometries have been reported for 4-MDBT and 4,6-DMDBT.

An analysis of the global minima of the molecular electrostatic potential (MEP) [58], $V_{\min }$, shows the most reactive sites of DBT, which correspond to the $\pi$-electron regions of the sulfur atom, as we show in Figure 1(a) and Table 1; the isosurfaces at constant $V(-55.79 \mathrm{~kJ} / \mathrm{mol})$ contain the positions of the global minima $V_{\min }$. For 4-MDBT and 4,6-DMDBT, the global minima are not located near the sulfur atoms, but at the $\pi$-electron regions of carbons in the aromatic rings, as we show in the isosurfaces of 4MDBT and 4,6-DMDBT at constant $V(-60.39 \mathrm{~kJ} / \mathrm{mol})$ in Figures 1(b) and 1(c), respectively. The results for $V_{\min }$ are in agreement with experimental studies of adsorption of DBT, 4-MDBT, and 4,6-DMDBT on catalytic surfaces; compared to DBT, 4-MDBT and 4,6-DMDBT adsorb strongly through the $\pi$-electrons of the aromatic carbon atoms [59]. Local minima of $V$ at the $\pi$-electron regions of the sulfur atoms, $V_{S}$, for 4-DBT and 4,6-DMDBT show slightly increased values with respect to DBT (Table 1). While the $V_{S}$ values at the $\pi$-electron regions of the sulfur atoms slightly increase, the total dipole moment decreases strongly from $0.7993 \mathrm{D}$ in DBT to $0.4665 \mathrm{D}$ and $0.0185 \mathrm{D}$ in 4 -MDBT and 4,6DMDBT, respectively (Table 1). The small dipole moment of 4,6-DMDBT (compared to the dipole moments of DBT and 4-MDBT) results in a much weaker attraction to the catalytic surface, thereby reducing the effectiveness of HDS and DDS processes [60].

The low rate for absorption and transformation of 4MDBT and 4,6-DMDBT at the HDS and DDS catalytic surfaces is related to the spatial "shielding" of the sulfur atom. The "shielding" results from the addition of methyl groups to DBT that reduce accessibility of the sulfur atom to catalytic surfaces, as shown in Figure 1. Accordingly, the small amount of 4-MDBT and 4,6-DMDBT transformed at HDS and DDS catalytic surfaces is probably done at spatially favorable sites, that is, at edges and defects. New approaches 
TABLE 1: Global minimum values of the molecular electrostatic potential $\left(V_{\min }\right)$ and local minimum values near the sulfur atoms $\left(V_{S}\right)$. Dipole moment, $\mu$, of DBT and its alkyl derivates using B3LYP/6-311+G(d, p).

\begin{tabular}{lccc}
\hline & $V_{\min }(\mathrm{kJ} / \mathrm{mol})$ & $V_{S}(\mathrm{~kJ} / \mathrm{mol})$ & $\mu(\mathrm{D})$ \\
\hline DBT & -60.65 & -60.65 & 0.7993 \\
4-MDBT & -61.96 & -60.91 & 0.4665 \\
$4,6-D M D B T$ & -64.06 & -61.17 & 0.0185 \\
\hline
\end{tabular}

for desulfurization processes should take into account these steric constraints, as well as the differences in their MEP and dipole moment to develop efficient and selective methods, regardless of the desulfurization mechanism used to separate these molecules from oil and its derived liquid fuels.

\section{Electronic Structure of the Monomeric Adsorbent}

To identify effective adsorbents, we need to quantify the monomer properties similar to the evaluation of organosulfur compound properties. Appropriate adsorbents for the organosulfur compounds should contain functional groups, which attract and form physical bonds with the sulfur atom. In a previous study, Castro et al. [61] proposed a structure containing secondary amines, which adsorb an oxidized product of DBT (sulfone). They found that the oxygen atoms of the sulfone interact with the hydrogen atoms of the secondary amines through hydrogen bonds. In this work, we propose a monomer structure that contains two primary amino groups and one hydroxyl group. We expect that the functional groups in the monomer will form hydrogen bonds with the sulfur atom in the organosulfur molecules. Specifically, we examine the monomeric structure shown in Figure 2 (named $\mathrm{M}$, for short). $\mathrm{M}$ can be synthesized through the ringopening reaction of an epoxide (2-propenoic acid, 2-methyl-, oxiranyl ester) with a pteridine containing a hydroxyl group (2,4-diamino-6-hydroxymethylpteridine) and an aluminum triflate as catalyst [62].

We calculate the optimized geometry and the energetics of the monomer at the same level of theory as the organosulfur compounds in the previous section. We also verify that the obtained conformation for the monomer corresponds to a global minimum with no imaginary frequencies. We map the structure of the monomer and its MEP to the electronic density isosurface of the molecule (Figure 2). The favorable sites of the monomer to interact with organosulfur compounds are revealed in the MEP map of the monomer. Electrondeficient sites in the monomer will attract negatively charged sites in the organosulfur compounds; those electron-deficient sites are primarily the regions containing the hydrogen of the hydroxyl group but they also occur in the regions containing the hydrogen atoms of the amino groups. Negatively charged sites in the monomer will attract electron-deficient sites in the organosulfur compounds. The regions around one of the central carbon atoms and its neighbor nitrogen atoms forming the double ring are negatively charged sites.

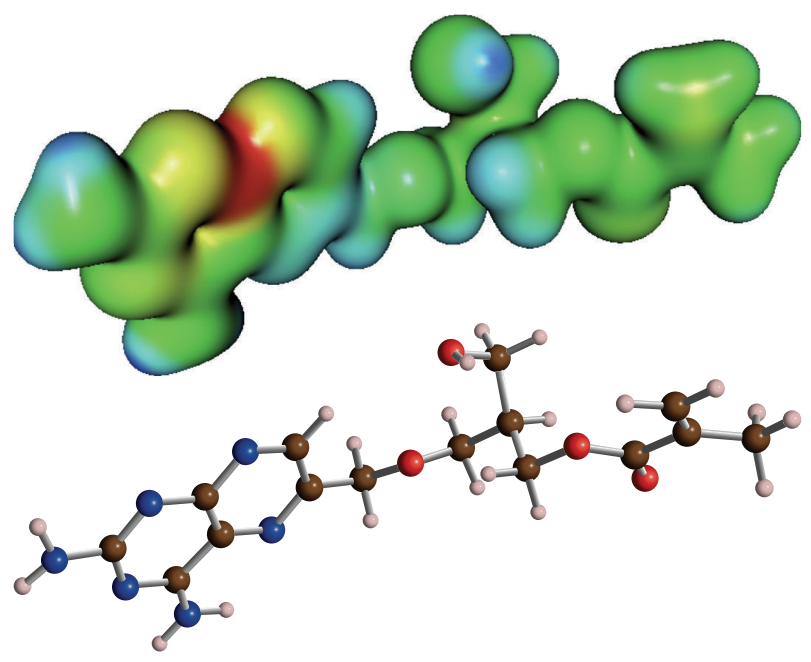

FIgURE 2: The molecular structure (bottom) and MEP mapped to the electronic density surface (isovalue $=0.02$ ) of monomer M (top). Brown spheres in the molecular structure represent carbon atoms, while white, red, and blue spheres represent hydrogen, oxygen, and nitrogen atoms, respectively. Blue areas in the MEP represent regions where a proton would feel repulsion, while in red areas a proton would feel attraction. Comparison of Figures 1 and 2 provides insight into which regions between the DBT series and $M$ will be attracted.

\section{Complexation Energies and Selectivities of DBT, 4-MDBT, and 4,6-DMDBT on $M$ and Its Derivates}

The stability of the complexes between DBT, 4-MDBT, and 4,6-DMDBT and the monomer $\mathrm{M}$ provides thermodynamic insight into the effectiveness of this monomer to adsorb these problematic organosulfur molecules. We optimize the geometries of the complexes at the same level of theory as the DBTs and M in Sections 3 and 4. For the complex with DBT, the organosulfur molecule was located initially with the sulfur atom near the amino groups of $\mathrm{M}$, but the optimized configuration resulted in a complex with a hydrogen bond between the sulfur atom and the hydrogen of the hydroxyl group of $\mathrm{M}$. We tested three other possible initial orientations, all of which resulted in the same final structure. Vibrational calculations show that the conformations obtained are global minimums with no imaginary frequencies. The organosulfur molecule also attaches to $M$ through hydrogen bonds between hydrogen atoms of DBT and a nitrogen atom in the double aromatic ring (Figure 3(a)). For the complexes with 4-MDBT and 4,6-DMDBT the separation between the sulfur atom and the hydrogen atoms of $\mathrm{M}$ becomes larger, probably due to the fact that the oxygen in the hydroxyl group and one of the nitrogen atoms in the double aromatic ring form hydrogen bonds with hydrogen atoms of the substituted methyl groups on 4-MDBT and 4,6-DMDBT. The hydrogen bonds with the methyl groups of the organosulfur compounds push the sulfur atom away from the monomeric structure. Figures 3(b) and 3(c) show snapshots of the optimized structures of the complexes with 4-MDBT and 4,6-DMDBT, respectively. 


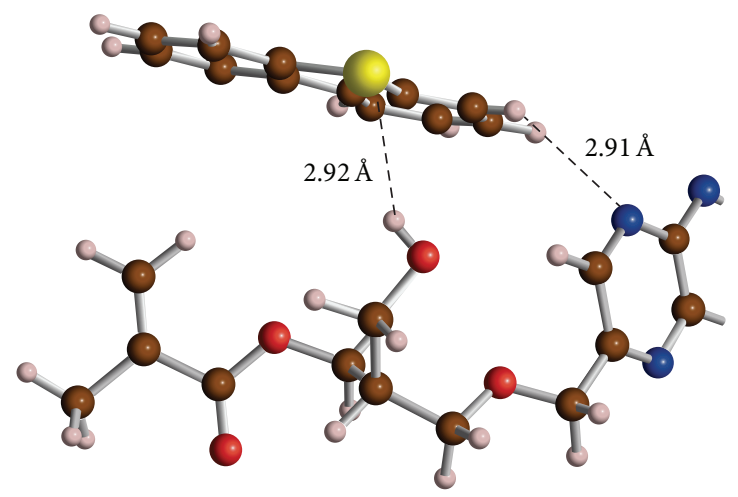

(a)

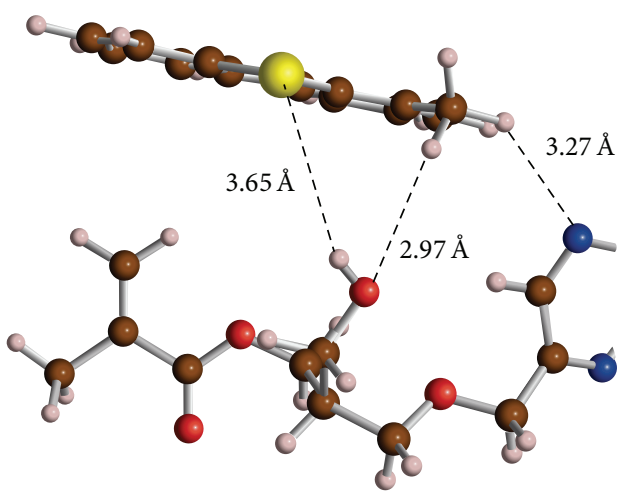

(b)

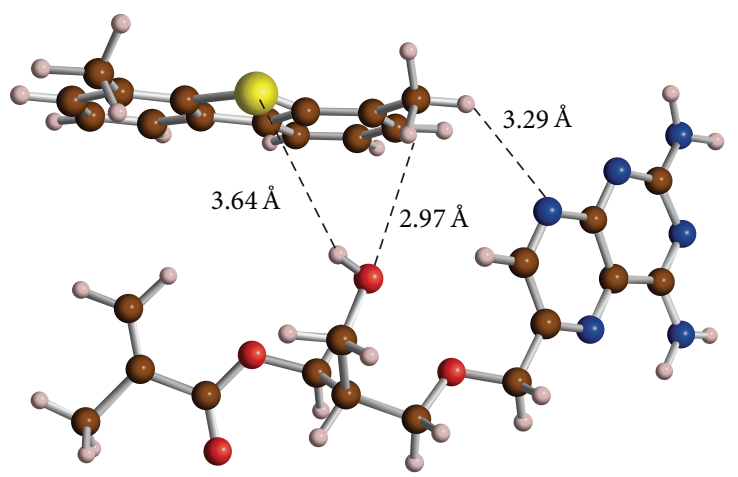

(c)

FIGURE 3: Structures of the complexes formed by (a) DBT, (b) 4-MDBT, and (c) 4,6-DMDBT with M. The organosulfur molecules are drawn at the top of each figure and we show only the part of M interacting with the organosulfur molecule. Labeled distances represent the hydrogen bonds between the organosulfur molecules and M. Atoms color codes of the molecular structures as in Figures 1 and 2.

We calculate the complexation energies including the zero-point correction by

$$
\Delta E_{\mathrm{ZPE}}=E_{\mathrm{ZPE}, \text { complex }}-\left(E_{\mathrm{ZPE}, \mathrm{M}}+E_{\mathrm{ZPE}, S}\right)
$$

where $E_{\mathrm{ZPE}, \mathrm{M}}, E_{\mathrm{ZPE}, S}$, and $E_{\mathrm{ZPE}, \text { complex }}$ are the total energies including the zero-point correction of the isolated monomer, the isolated organosulfur compound, and the complex, respectively. We calculate the total energies including the zero-point correction of the complex and the isolated molecules through vibrational calculations, using the previously optimized structures at the same level of theory used for the isolated constituents. At $0 \mathrm{~K}$, the free energy, $\Delta G^{0 \mathrm{~K}}$, is equal to the change in enthalpy and also is equal to $\Delta E_{\mathrm{ZPE}}$. The $\Delta E_{\mathrm{ZPE}}$ for the 3 complexes between $\mathrm{M}$ and $\mathrm{DBT}, \mathrm{M}$ and 4-MDBT, and $\mathrm{M}$ and 4,6-DMDBT show negative values indicating exothermic and spontaneous adsorption processes at $0 \mathrm{~K}$. The complex with 4,6 -DMDBT shows the largest value, while the complex with DBT shows the lowest value (Table 2). The observed trend in energies is probably due to the additional hydrogen bonds between the hydrogen atoms of the methyl substituent and $\mathrm{M}$, which are not present in the complex with DBT. We repeat the vibrational calculations at $300 \mathrm{~K}$ and $1 \mathrm{~atm}$ and find that at these thermodynamic conditions the process starting from isolated reactants is exothermic but not spontaneous.
We calculate the selectivity $\left(\alpha_{i}\right)$ of component $i$ with respect to DBT using the complexation free energies and the criteria

$$
\alpha_{i}=\Delta G_{i}^{0 \mathrm{~K}}-\Delta G_{\mathrm{DBT}}^{0 \mathrm{~K}}
$$

where $\Delta G_{i}^{0 \mathrm{~K}}$ and $\Delta G_{\mathrm{DBT}}^{0 \mathrm{~K}}$ are the free energies at $0 \mathrm{~K}$ of component $i$ and DBT, respectively. The selectivities of 4-MDBT and 4,6-DMDBT with reference to DBT show negative values (Table 3), indicating that 4-MDBT and 4,6-DMDBT will be preferably adsorbed compared to DBT, but the selectivities can change depending upon the thermodynamic conditions and solvents surrounding the complexes and molecules. Therefore we study how the structure of the monomer affects $\alpha_{i}$. In order to improve $\alpha_{i}$ towards the problematic compounds (4-MDBT and 4,6-DMDBT), we modify the structure of M. Since the sulfur atom forms hydrogen bonds with hydroxyl and amino groups and hydrogen atoms attached to aromatic rings [63], we affect $\alpha_{i}$ by making the hydrogen of the hydroxyl group more or less accessible to the sulfur atom. We build five new monomer structures of $M$ by replacing the methylene group (and its attached hydroxyl functional group) with alcohol chains having no methylene groups (which we refer to as $\mathrm{M}_{0}$ ) and alcohol chains with up to five methylene groups (we call these $\mathrm{M}_{2}-\mathrm{M}_{5}$ ) as shown in Figure 4. 


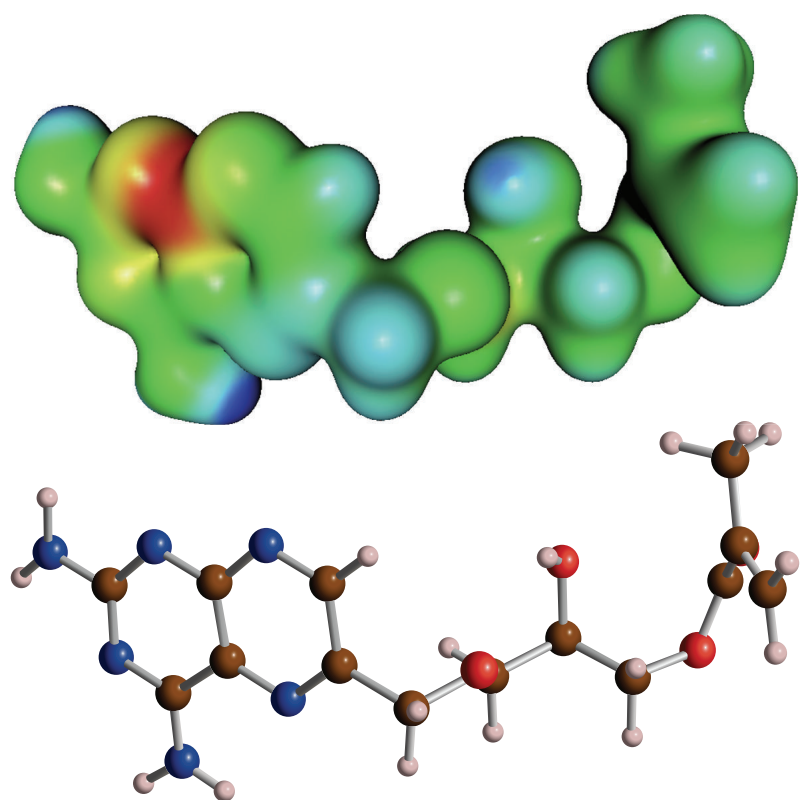

(a)

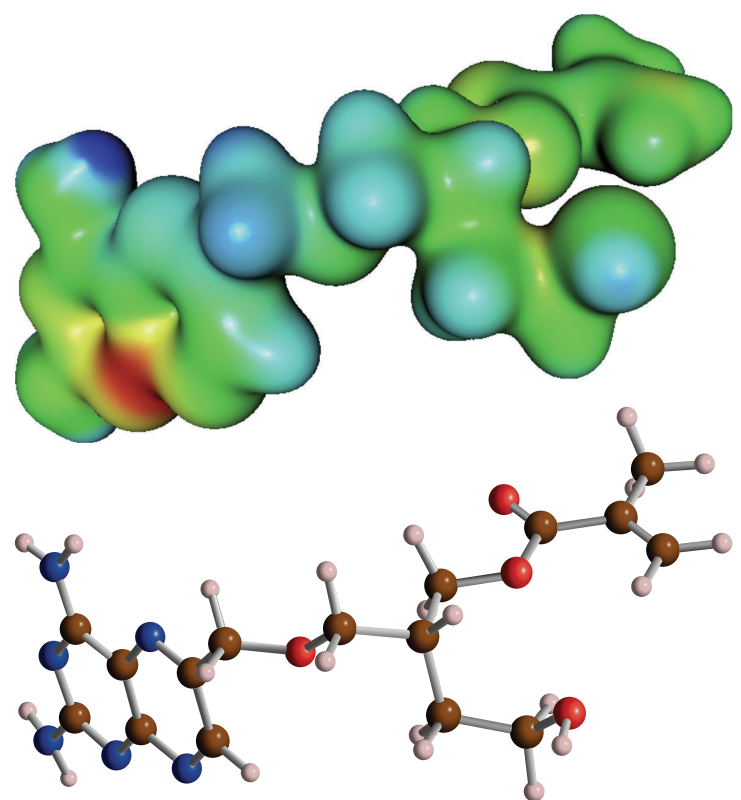

(b)

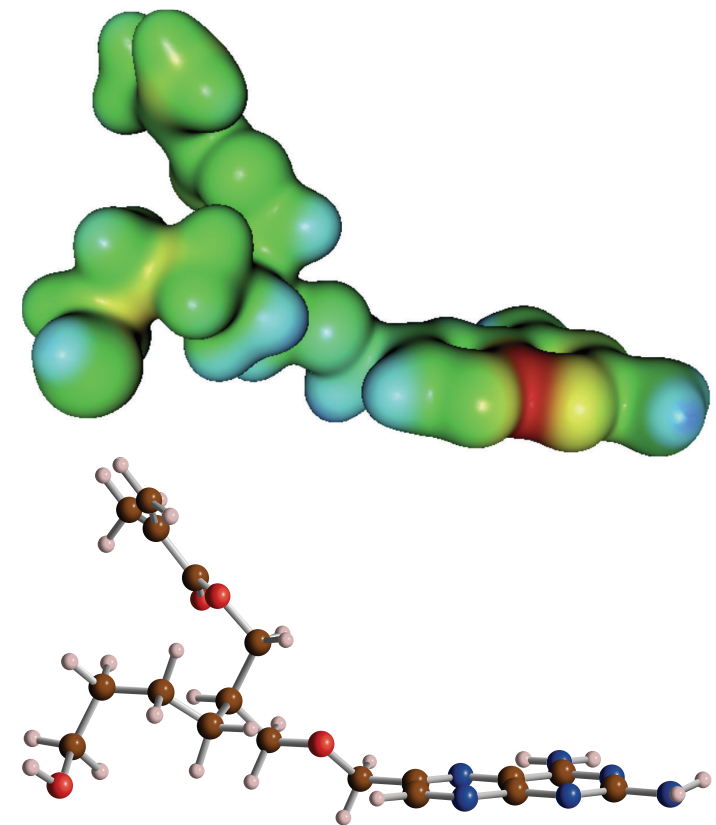

(c)

Figure 4: Molecular structure (bottom) and MEP (top) mapped to the electronic density surface (isovalue $=0.02$ ) of monomers (a) $\mathrm{M}_{0}$, (b) $\mathrm{M}_{2}$, and (c) $\mathrm{M}_{4}$. Color codes as in Figures 1 and 2.

We optimize the structures of the complexes and calculate $\Delta E_{\mathrm{ZPE}}$ and $\alpha_{i}$ of the complexes formed by DBT, 4-MDBT, and 4,6-DMDBT with the five derivates of $\mathrm{M}\left(\mathrm{M}_{0}, \mathrm{M}_{2}-\mathrm{M}_{5}\right)$ at the same level of theory used for the complexes with $\mathrm{M}$. Vibrational calculations using the optimized conformation of the complexes show global minimums with no imaginary frequencies. We calculate $\Delta E_{\mathrm{ZPE}}$ and $\alpha_{i}$ for all the complexes with (1) and (2) and report the values in Tables 2 and 3 and in Figure 5. In order to validate the complexation energy results, we also perform single-point energy calculations for the complexes with 4,6-DMDBT and $\mathrm{M}_{3}-\mathrm{M}_{5}$ at the more accurate method MP2 using a smaller basis set (6-311G), using conformations optimized with B3LYP at the same basis set, and we also report the complexation energy results in Table 2. We were unable to use MP2 with the $6-311+G(d, p)$ basis set due to computational limitations. The MP2 results do not include the zero-point energy correction also due to computational limitations. We find that MP2 and B3LYP 
TABLE 2: Complexation energies including zero-point correction for DBT, 4-MDBT, and 4,6-DMDBT on monomeric structures $\mathrm{M}_{0}-\mathrm{M}_{5}$ using B3LYP/6-311+G(d, p).

\begin{tabular}{lccc}
\hline Monomer & $\Delta E_{\mathrm{ZPE}, \mathrm{DBT}}(\mathrm{kJ} / \mathrm{mol})$ & $\Delta E_{\mathrm{ZPE}, 4-\mathrm{MDBT}}(\mathrm{kJ} / \mathrm{mol})$ & $\Delta E_{\mathrm{ZPE}, 4,6-\mathrm{DMDBT}}(\mathrm{kJ} / \mathrm{mol})^{\mathrm{a}}$ \\
\hline $\mathrm{M}_{0}$ & -15.11 & -13.22 & -9.52 \\
$\mathrm{M}_{1}(\mathrm{M})$ & -14.11 & -14.54 & -14.78 \\
$\mathrm{M}_{2}$ & -9.51 & -10.38 & -9.69 \\
$\mathrm{M}_{3}$ & -10.50 & -23.14 & $-10.02(-61.34)$ \\
$\mathrm{M}_{4}$ & -8.72 & -9.83 & $-9.79(-53.41)$ \\
$\mathrm{M}_{5}$ & -12.90 & -13.49 & $-13.05(-63.02)$ \\
\hline
\end{tabular}

${ }^{a}$ Values in parentheses represent results for geometry optimizations at the B3LYP/6-311G level of theory and complexation energies from single-point energy calculations at the MP2/6-311G level of theory.

TABLE 3: Selectivity of 4-MDBT and 4,6-DMDBT with reference to DBT on monomeric structures $\mathrm{M}_{0}-\mathrm{M}_{5}$ calculated using (2).

\begin{tabular}{lcc}
\hline Monomer & $\alpha_{4-\mathrm{MDBT}}$ & $\alpha_{4,6-\mathrm{DMDBT}}$ \\
\hline $\mathrm{M}_{0}$ & 1.89 & 5.59 \\
$\mathrm{M}_{1}(\mathrm{M})$ & -0.43 & -0.67 \\
$\mathrm{M}_{2}$ & -0.87 & -0.18 \\
$\mathrm{M}_{3}$ & -12.65 & 0.47 \\
$\mathrm{M}_{4}$ & -1.10 & -1.07 \\
$\mathrm{M}_{5}$ & -0.58 & -0.15 \\
\hline
\end{tabular}

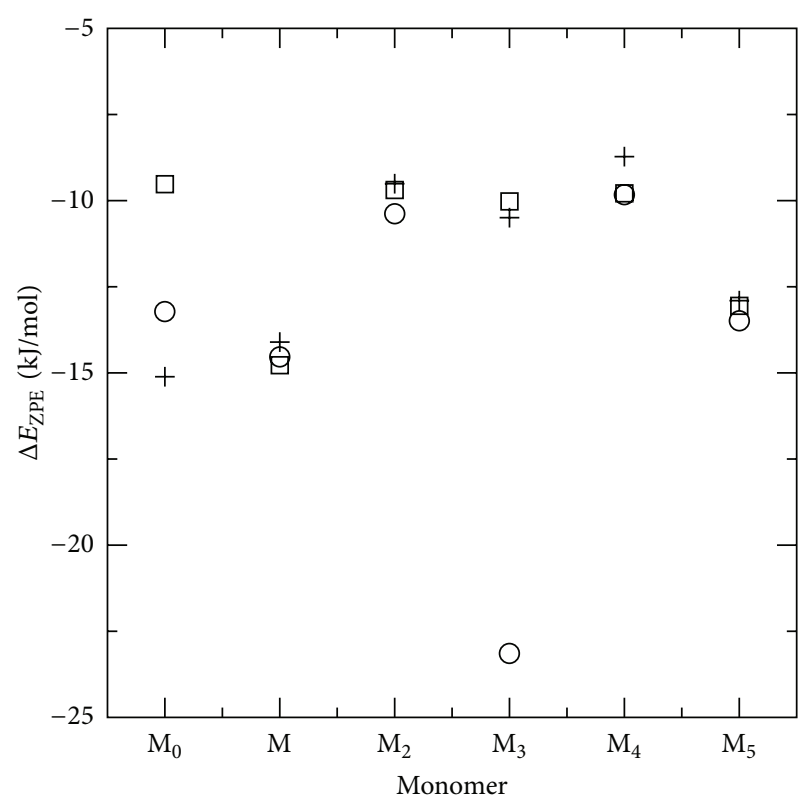

FIGURE 5: Complexation energies including zero-point correction of DBT, 4-MDBT, and 4,6-DMDBT on monomers $\mathrm{M}_{0}-\mathrm{M}_{5}$. Plus symbols represent the complexes with DBT, circles represent the complexes with 4-MDBT, and squares represent the complexes with 4,6-DMDBT.

produce a similar trend, but the MP2 results show larger values, probably due to the basis set used to optimize the geometries and to calculate the energy values.

The largest $\Delta G^{0 \mathrm{~K}}\left(=\Delta E_{\mathrm{ZPE}}\right)$ correspond to the complexes formed between DBT- $\mathrm{M}_{0}, 4-\mathrm{DMDBT}-\mathrm{M}_{3}$, and 4,6DMDBT-M, but the highest $\alpha_{i}$ correspond to the complexes

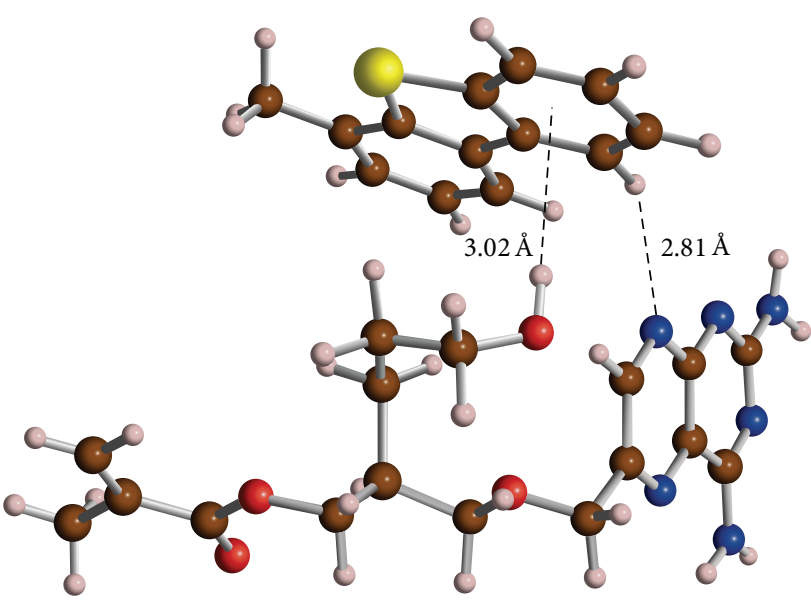

FIGURE 6: Molecular structures of the complexes formed by 4MDBT and $\mathrm{M}_{3}$. The organosulfur molecules are drawn at the top. Color codes as in Figures 1 and 2. One of the labeled distances represent the hydrogen bond between the hydrogen of the hydroxyl group of $\mathrm{M}_{3}$ and the center of mass of one of the aromatic rings of 4MDBT. Also we label the distance of the hydrogen bond between one of the hydrogen atoms of 4-MDBT and one of the nitrogen atoms of $\mathrm{M}_{3}$.

4-MDBT-M 3 and 4,6-DMDBT-M $\mathrm{M}_{4}$. Unfortunately, 4-MDBT molecules adsorb on $\mathrm{M}_{3}$ through the atoms of one of the aromatic rings and hydrogen bonds between the sulfur atom and the hydrogen of the hydroxyl group are not formed (Figure 6). Many organic compounds with no sulfur atoms will form stable complexes and show hydrogen bonds with $\mathrm{M}_{3}$ similar to the bonds between 4-MDBT and $\mathrm{M}_{3}$. Those complexes will have similar complexation free energies and therefore the selectivity will be similar if we do not take into account the solvent effects [64]. Hence we must investigate other monomer structures with smaller $\alpha_{4 \text {-MDBT }}$ to see if they might be more effective.

The complexes formed by the organosulfur compounds and $\mathrm{M}_{4}$ include hydrogen bonds between the sulfur atom and the hydrogen of the hydroxyl group (Figure 7). The complexes of 4-MDBT and 4,6-DMDBT and $\mathrm{M}_{4}$ also show additional hydrogen bonds between the hydrogen atoms of the methyl substituent of the organosulfur molecule and the oxygen of the hydroxyl group of $\mathrm{M}_{4}$. The distance of the additional hydrogen bonds is $\sim 2.72 \AA$, revealing strong 


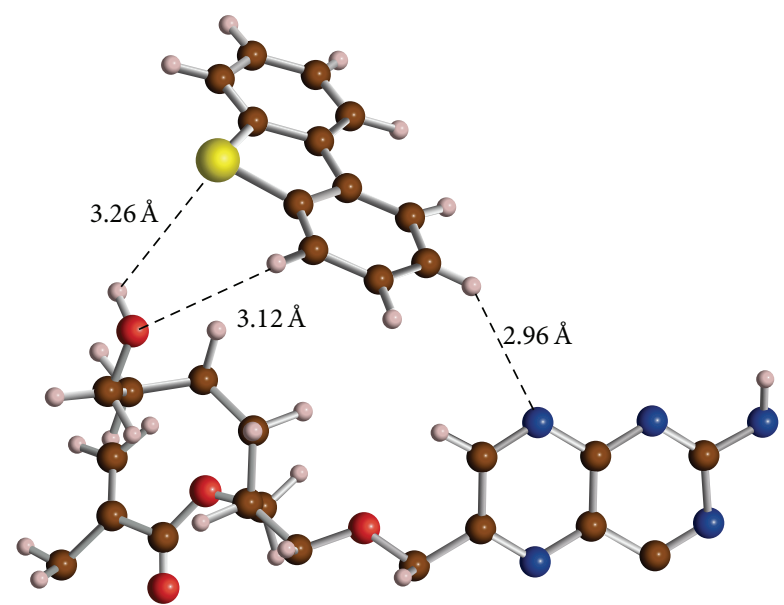

(a)

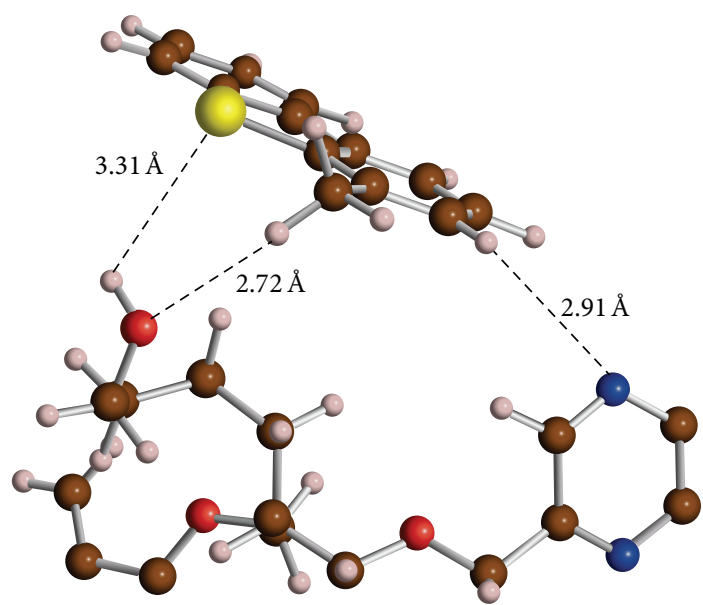

(b)

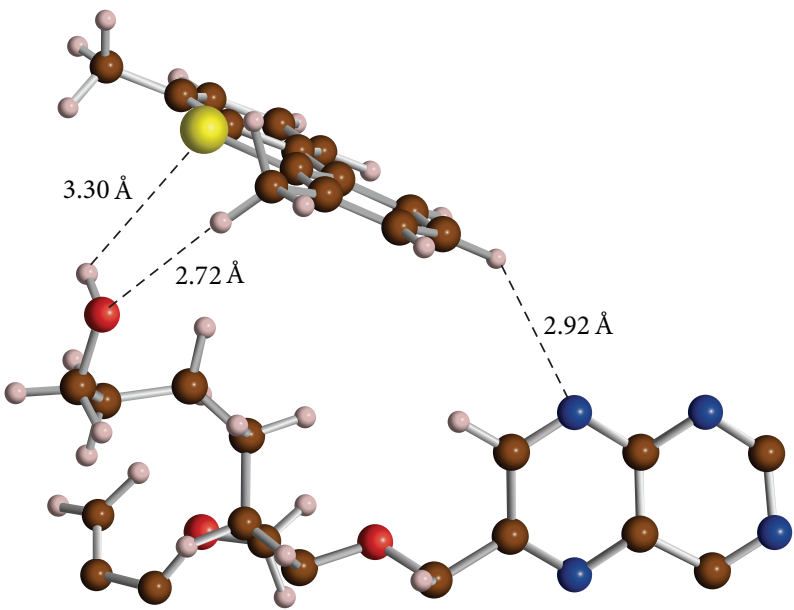

(c)

Figure 7: Molecular structures of the complexes formed by (a) DBT, (b) 4-MDBT, and (c) 4,6-DMDBT with $\mathrm{M}_{4}$. The organosulfur molecules are drawn at the top of each figure and we show only the part of $\mathrm{M}_{4}$ interacting with the organosulfur molecule. Color codes as in Figures 1 and 2. Labeled distances represent hydrogen bonds between the organosulfur molecules and $\mathrm{M}_{4}$.

hydrogen bonds. The complexation energies on monomer $\mathrm{M}_{4}$ are similar to previously reported DFT calculations of the adsorption of thiophene on clusters representing zeolites (6.1-10.5 kJ/mol) [65]. Experimental adsorption of DBT, 4MDBT, and 4,6-DMDBT on activated carbons shows that selectivities towards the problematic compounds 4-MDBT, between 1.28 and 2.3, and 4,6-DMDBT, between 1.96 and 3.50, using DBT as reference [66]. The activated carbons probably have active sites (hydroxyl and carboxylic) that interact with 4-MDBT and 4,6-DMBT in a similar way as those studied in this work. If we consider the selectivities and the need for complexes forming bonds with the sulfur atom, $\mathrm{M}_{4}$ is a better adsorbent than $\mathrm{M}_{3}$ to process the problematic organosulfur compounds 4-MDBT and 4,6-DMDBT.

\section{Conclusions}

We have shown that polymeric materials with structures including amino and hydroxyl functional groups form stable complexes with dibenzothiophene and its alkyl derivates.
The complexation energies at $0 \mathrm{~K}$ for the complexes formed between organosulfur molecules and monomeric structures of these polymers show spontaneous and exothermic processes for all monomeric structures studied.

The best monomeric structure $\left(\mathrm{M}_{4}\right)$ found in this work for the selectivity of 4-MDBT and 4,6-DMDBT has a hydroxyl group attached to a linear chain long enough to produce a hydrogen bond between the sulfur atom of the organosulfur molecule and the hydrogen of the hydroxyl group. The linear chain studied in this work has the appropriate length to allow for other hydrogen bonds between the hydrogen atoms of the methyl substituent of the organosulfur molecule and the nitrogen atoms of the monomeric structure.

Additional calculations are needed to account for solvent effects to produce reliable selectivity predictions. A relevant solvent for these systems is a model solution of oil or its fractions, which are difficult to study using the basis set used in this work. Therefore, calculations using less expensive computational resources are needed. Nonetheless, our initial results at $0 \mathrm{~K}$ show that these monomeric structures are 
a possible solution to eliminate these pollutants from oil and its derivates.

In future work, a study of the selectivity of the adsorption of the problematic organosulfur molecules using the best monomeric structures found in this work in a polymeric system containing several monomer units using molecular dynamics simulations will be conducted to study the effectiveness of these systems in more realistic conditions. The polymeric chain will be in contact with mixtures of oil, gasoline, and diesel containing representative amounts of organosulfur compounds.

\section{Conflict of Interests}

The authors declare that there is no conflict of interests regarding the publication of this paper.

\section{Acknowledgments}

Jose L. Rivera thanks Universidad Michoacana de San Nicolás de Hidalgo (México) and CONACYT (México) for support under Grant no. 134508. Pedro Navarro-Santos thanks CONACYT (México) for the postdoctoral fellowship under Grant no. 170026. Roberto Guerra-Gonzalez thanks PROMEP (Mexico) for financial assistance under Grant no. UMSNH-EXB-223.

\section{References}

[1] R. A. Corbett, "Tougher diesel specs could force major refining industry expenditures," Oil and Gas Journal, vol. 85, p. 56, 1987.

[2] M. R. Khan and J. G. Reynolds, "Formulating a response to the clean air act," Chemtech, vol. 26, no. 6, pp. 56-61, 1996.

[3] E. Ito and J. A. R. van Veen, "On novel processes for removing sulphur from refinery streams," Catalysis Today, vol. 116, no. 4, pp. 446-460, 2006.

[4] C. Song, "An overview of new approaches to deep desulfurization for ultra-clean gasoline, diesel fuel and jet fuel," Catalysis Today, vol. 86, pp. 211-263, 2003.

[5] C. Song and X. L. Ma, "New design approaches to ultra-clean diesel fuels by deep desulfurization and deep dearomatization," Applied Catalysis B: Environmental, vol. 41, no. 1-2, pp. 207-238, 2003.

[6] C. S. Song and X. L. Ma, "Ultra-deep desulfurization of liquid hydrocarbon fuels: chemistry and process," International Journal of Green Energy, vol. 1, no. 2, pp. 167-191, 2004.

[7] H. Rang, J. Kann, and V. Oja, "Advances in desulfurization research of liquid fuel," Oil Shale, vol. 23, no. 2, pp. 164-176, 2006.

[8] A. M. Fonseca, J. L. Biscaya, J. Aires-de-Sousa, and A. M. Lobo, "Geographical classification of crude oils by Kohonen self-organizing maps," Analytica Chimica Acta, vol. 556, no. 2, pp. 374-382, 2006.

[9] E. E. Donath and M. Hoering, "Early coal hydrogenation catalysis," Fuel Processing Technology, vol. 1, no. 1, pp. 3-20, 1977.

[10] H. Topsøe, B. S. Clausen, and F. E. Massoth, Hydrotreating Catalysis Science and Technology, Springer, Berlin, Germany, 1996.

[11] V. Lamure-Meille, E. Schulz, M. Lemaire, and M. Vrinat, "Effect of experimental parameters on the relative reactivity of dibenzothiophene and 4-methyldibenzothiophene," Applied Catalysis A: General, vol. 131, no. 1, pp. 143-157, 1995.

[12] R. Shafi and G. J. Hutchings, "Hydrodesulfurization of hindered dibenzothiophenes: an overview," Catalysis Today, vol. 59, no. 3, pp. 423-442, 2000.

[13] J. T. Sampanthar, H. Xiao, J. Dou, T. Y. Nah, X. Rong, and W. P. Kwan, "A novel oxidative desulfurization process to remove refractory sulfur compounds from diesel fuel," Applied Catalysis B: Environmental, vol. 63, no. 1-2, pp. 85-93, 2006.

[14] B. C. Gates and H. Topsøe, "Reactivities in deep catalytic hydrodesulfurization: challenges, opportunities, and the importance of 4-methyldibenzothiophene and 4,6-dimethyldibenzothiophene," Polyhedron, vol. 16, no. 18, pp. 3213-3217, 1997.

[15] I. B. W. Gunam, Y. Yaku, M. Hirano et al., "Biodesulfurization of alkylated forms of dibenzothiophene and benzothiophene by Sphingomonas subarctica T7b," Journal of Bioscience and Bioengineering, vol. 101, no. 4, pp. 322-327, 2006.

[16] L. Rashidi, G. Mohebali, J. T. Darian, and B. Rasekh, "Biodesulfurization of dibenzothiophene and its alkylated derivatives through the sulfur-specific pathway by the bacterium RIPI-S81," African Journal of Biotechnology, vol. 5, no. 4, pp. 351-356, 2006.

[17] S. Guobin, Z. Huaiying, C. Weiquan, X. Jianmin, and L. Huizhou, "Improvement of biodesulfurization rate by assembling nanosorbents on the surfaces of microbial cells," Biophysical Journal, vol. 89, no. 6, pp. L58-L60, 2005.

[18] J. J. Lee, H. Kim, and S. H. Moon, "Preparation of highly loaded, dispersed $\mathrm{MoS}_{2} / \mathrm{Al}_{2} \mathrm{O}_{3}$ catalysts for the deep hydrodesulfurization of dibenzothiophenes," Applied Catalysis B: Environmental, vol. 41, no. 1-2, pp. 171-180, 2003.

[19] H. Y. Kim, J. J. Lee, J. H. Koh, and S. H. Moon, "Performance of fluorine-added, sonochemically prepared $\mathrm{MoS}_{2} / \mathrm{Al}_{2} \mathrm{O}_{3}$ catalysts in the hydrodesulfurization of dibenzothiophene compounds," Applied Catalysis B: Environmental, vol. 54, no. 1, pp. 33-39, 2004.

[20] I. K. Kim and C. P. Huang, "Sonochemical degradation of polycyclic aromatic sulfur hydrocarbons (PASHs) in aqueous solutions exemplified by benzothiophene," Journal of the Chinese Institute of Engineers, vol. 28, no. 7, pp. 1107-1118, 2005.

[21] A. Milenkovic, E. Schulz, V. Meille et al., "Selective elimination of alkyldibenzothiophenes from gas oil by formation of insoluble charge-transfer complexes," Energy and Fuels, vol. 13, no. 4, pp. 881-887, 1999.

[22] T. Koltai, M. Macaud, A. Milenkovic, E. Schulz, M. Lemaire, and M. Vrinat, "Hydrodesulfurization of diesel feeds by association of a catalytic process and a separation process using chargetransfer complexes," Catalysis Letters, vol. 83, no. 3-4, pp. 143148, 2002.

[23] A. Milenkovic, D. Loffreda, E. Schulz, H. Chermette, M. Lemaire, and P. Sautet, "Charge transfer complexes between tetranitrofluorenone and polyaromatic compounds from gasoil: a combined DFT and experimental study," Physical Chemistry Chemical Physics, vol. 6, no. 6, pp. 1169-1180, 2004.

[24] G. B. Shan, H. Y. Zhang, H. Z. Liu, and J. M. Xing, " $\pi$ complexation studied by fluorescence technique: application in desulfurization of petroleum product using magnetic $\pi$ complexation sorbents," Separation Science and Technology, vol. 40, no. 14, pp. 2987-2999, 2005.

[25] S. Velu, X. L. Ma, and C. S. Song, "Selective adsorption for removing sulfur from jet fuel over zeolite-based adsorbents," Industrial and Engineering Chemistry Research, vol. 42, no. 21, pp. 5293-5304, 2003. 
[26] D. H. Zhou, Y. Q. Wang, N. He, and G. Yang, "The $\pi$ complexation mechanisms of $\mathrm{Cu}(\mathrm{I}), \mathrm{Ag}(\mathrm{I}) /$ zeolites for desulfurization," Acta Physico-Chimica Sinica, vol. 22, no. 5, pp. 542-547, 2006.

[27] S. G. McKinley and R. J. Angelici, "Deep desulfurization by selective adsorption of dibenzothiophenes on $\mathrm{Ag}^{+} / \mathrm{SBA}-15$ and $\mathrm{Ag}^{+} / \mathrm{SiO}_{2}$," Chemical Communications, vol. 9, no. 20, pp. 26202621, 2003.

[28] S. Haji and C. Erkey, "Removal of dibenzothiophene from model diesel by adsorption on carbon aerogels for fuel cell applications," Industrial and Engineering Chemistry Research, vol. 42, no. 26, pp. 6933-6937, 2003.

[29] X. Sun, B. Xu, T. Xiao, Q. Zha, and F. M. Alshahrani, "Adsorptive desulfurization using carbon materials with different surface areas," Progress in Natural Science, vol. 15, pp. 105-110, 2005.

[30] D. Jayne, Y. Zhang, S. Haji, and C. Erkey, "Dynamics of removal of organosulfur compounds from diesel by adsorption on carbon aerogels for fuel cell applications," International Journal of Hydrogen Energy, vol. 30, no. 11, pp. 1287-1293, 2005.

[31] J. Aburto and S. le Borgne, "Selective adsorption of dibenzothiophene sulfone by an imprinted and stimuli-responsive chitosan hydrogel," Macromolecules, vol. 37, no. 8, pp. 2938-2943, 2004.

[32] J. Aburto, A. Mendez-Orozco, and S. le Borgne, "Hydrogels as adsorbents of organosulphur compounds currently found in diesel," Chemical Engineering and Processing: Process Intensification, vol. 43, no. 12, pp. 1587-1595, 2004.

[33] S. H. D. Lee, R. Kumar, and M. Krumpelt, "Sulfur removal from diesel fuel-contaminated methanol," Separation and Purification Technology, vol. 26, no. 2-3, pp. 247-258, 2002.

[34] C. O. Ania and T. J. Bandosz, "Importance of structural and chemical heterogeneity of activated carbon surfaces for adsorption of dibenzothiophene," Langmuir, vol. 21, no. 17, pp. 7752-7759, 2005.

[35] C. O. Ania and T. J. Bandosz, "Sodium on the surface activated carbons as a factor enhancing reactive adsorption of dibenzothiophene," Energy and Fuels, vol. 20, no. 3, pp. 1076-1080, 2006.

[36] A. V. Morozov, T. Kortemme, K. Tsemekhman, and D. Baker, "Close agreement between the orientation dependence of hydrogen bonds observed in protein structures and quantum mechanical calculations," Proceedings of the National Academy of Sciences of the United States of America, vol. 101, no. 18, pp. 6496-6951, 2004.

[37] G. A. Kumar, Y. Pan, C. J. Smallwood, and M. A. McAllister, "Low-barrier hydrogen bonds: Ab initio and DFT investigation," Journal of Computational Chemistry, vol. 19, no. 12, pp. 1345-1352, 1998.

[38] J. A. Dobado and J. Molina, "Adenine-hydrogen peroxide system: DFT and MP2 investigation," Journal of Physical Chemistry A, vol. 103, no. 24, pp. 4755-4761, 1999.

[39] M. J. Frisch, G. W. Trucks, H. B. Schlegel et al., Gaussian 03, Revision C. 02, Gaussian, Wallingford CT, USA, 2004.

[40] A. D. Becke, "A new mixing of Hartree-Fock and localdensityfunctional theories," The Journal of Chemical Physics, vol. 98, no. 2, p. 1372, 1993.

[41] C. Lee, W. Yang, and R. G. Parr, "Development of the ColleSalvetti correlation-energy formula into a functional of the electron density," Physical Review B, vol. 37, p. 785, 1988.

[42] M. A. McAllister, "Characterization of low-barrier hydrogen bonds $2 . \mathrm{HF}_{2}{ }^{-}$: a density functional and ab initio study," Journal of Molecular Structure: Theochem, vol. 427, pp. 39-53, 1998.
[43] J. R. B. Gomes and M. A. V. R. da Silva, "Thermochemistry of small organosulfur compounds from ab initio calculations," Journal of Physical Chemistry A, vol. 108, no. 52, pp. 11684-11690, 2004.

[44] V. L. S. Freitas, J. R. B. Gomes, and M. D. M. C. Ribeiro da Silva, "Revisiting dibenzothiophene thermochemical data: experimental and computational studies," Journal of Chemical Thermodynamics, vol. 41, no. 11, pp. 1199-1205, 2009.

[45] I. Garcia-Cruz, D. Valencia, T. Klimova et al., "Proton affinity of S-containing aromatic compounds: implications for crude oil hydrodesulfurization," Journal of Molecular Catalysis A: Chemical, vol. 281, no. 1-2, pp. 79-84, 2008.

[46] D. K. Singh, S. K. Srivastava, A. K. Ojha, and B. P. Asthana, "Vibrational study of thiophene and its solvation in two polar solvents, DMSO and methanol by Raman spectroscopy combined with ab initio and DFT calculations," Journal of Molecular Structure, vol. 892, pp. 384-391, 2008.

[47] R. Krishnan, J. S. Binkley, R. Seeger, and J. A. Pople, "Selfconsistent molecular orbital methods. XX. A basis set for correlated wave functions," The Journal of Chemical Physics, vol. 72, no. 1, pp. 650-654, 1980.

[48] C. H. Suresh and N. Koga, "Quantifying the electronic effect of substituted phosphine ligands via molecular electrostatic potential," Inorganic Chemistry, vol. 41, no. 6, pp. 1573-1578, 2002.

[49] X. Liu, G. Zhou, X. Zhang, and S. Zhang, "Molecular dynamics simulation of desulfurization by ionic liquids," AIChE Journal, vol. 56, no. 11, pp. 2983-2996, 2010.

[50] S. Jameh-Bozorghi and A. Niazi, "QSAR study of capacity factors by quantum chemical descriptorsand using PLS and LSSVM methods," Journal of Sciences (Islamic Azad University), vol. 20, no. 76, p. 101, 2010.

[51] H. Xu, Z. Han, D. Zhang, and J. Zhan, "Interface behaviors of acetylene and ethylene molecules with 1-butyl-3methylimidazolium acetate ionic liquid: a combined quantum chemistry calculation and molecular dynamics simulation study," ACS Applied Materials and Interfaces, vol. 12, no. 12, pp. 6646-6653, 2012.

[52] M. T. Feldmann, S. L. Widicus, G. A. Blake, D. R. Kent IV, and W. A. Goddard, "Aminomethanol water elimination: theoretical examination," Journal of Chemical Physics, vol. 123, no. 3, Article ID 034304, 2005.

[53] M. S. Rudner, S. Jeremic, K. A. Petterson et al., "Intramolecular hydrogen bonding in disubstituted ethanes. A comparison of $\mathrm{NH} \cdots \mathrm{O}^{-}$and $\mathrm{OH} \cdots \mathrm{O}^{-}$hydrogen bonding through conformational analysis of 4-Amino-4-oxobutanoate (succinamate) and monohydrogen 1,4-butanoate (monohydrogen succinate) anions," Journal of Physical Chemistry A, vol. 109, no. 40, pp. 9076-9082, 2005.

[54] S. S. Jang and W. A. Goddard III, "Structures and properties of newton black films characterized using molecular dynamics simulations," The Journal of Physical Chemistry B, vol. 110, no. 15, pp. 7992-8001, 2006.

[55] J. B. Foresman and A. Frisch, Exploring Chemistry with Electronic Structure Methods: A Guide Using Gaussian, Gaussian, Pittsburgh, Pa, USA, 2nd edition, 1995.

[56] F. M. Juárez-Guerra, J. L. Rivera, A. Zúñiga-Moreno, L. A. Galicia-Luna, J. L. Rico, and J. Lara, "Molecular modeling of thiophene in the vapor-liquid equilibrium," Separation Science and Technology, vol. 41, no. 2, pp. 261-281, 2006. 
[57] R. M. Schaffrin and J. Trotter, "Structure of dibenzothiophen," Journal of the Chemical Society A: Inorganic, Physical, and Theoretical Chemistry, pp. 1561-1565, 1970.

[58] C. H. Suresh, P. Alexander, K. P. Vijayalakshmi, P. K. Sajith, and S. R. Gadre, "Use of molecular electrostatic potential for quantitative assessment of inductive effect," Physical Chemistry Chemical Physics, vol. 10, no. 43, pp. 6492-6499, 2008.

[59] T. Kabe, A. Ishihara, and Q. Zhang, "Deep desulfurization of light oil. Part 2: hydrodesulfurization of dibenzothiophene, 4methyldibenzothiophene and 4,6-dimethyldibenzothiophene," Applied Catalysis A: General, vol. 97, no. 1, pp. L1-L9, 1993.

[60] G. R. Hutchison, M. A. Ratner, T. J. Marks, and R. Naaman, "Adsorption of polar molecules on a molecular surface," The Journal of Physical Chemistry B, vol. 105, no. 15, pp. 2881-2884, 2001.

[61] B. Castro, M. J. Whitcombe, E. N. Vulfson, R. Vazquez-Duhalt, and E. Bárzana, "Molecular imprinting for the selective adsorption of organosulphur compounds present in fuels," Analytica Chimica Acta, vol. 435, no. 1, pp. 83-90, 2001.

[62] D. Bradley, G. Williams, and M. Lawton, "Aluminium triflate: a remarkable Lewis acid catalyst for the ring opening of epoxides by alcohols," Organic and Biomolecular Chemistry, vol. 3, no. 18, pp. 3269-3272, 2005.

[63] F. H. Allen, C. M. Bird, R. S. Rowland, and P. R. Raithby, "Hydrogen-bond acceptor and donor properties of divalent sulfur (Y-S-Z and R-S-H)," Acta Crystallographica B: Structural Science, vol. 53, no. 4, pp. 696-701, 1997.

[64] A. J. Ruben, Y. Kiso, and E. Freire, "Overcoming roadblocks in lead optimization: a thermodynamic perspective," Chemical Biology and Drug Design, vol. 67, no. 1, pp. 2-4, 2006.

[65] H. Soscun, O. Castellano, J. Hernandez, and A. Hinchliffe, "Theoretical study of the structural, vibrational, and topologic properties of the charge distribution of the molecular complexes between thiophene and Brönsted acid sites of zeolites," International Journal of Quantum Chemistry, vol. 87, no. 4, pp. 240-253, 2002.

[66] A. Zhou, X. Ma, and C. Song, "Liquid-phase adsorption of multi-ring thiophenic sulfur compounds on carbon materials with different surface properties," The Journal of Physical Chemistry B, vol. 110, pp. 4699-4707, 2006. 

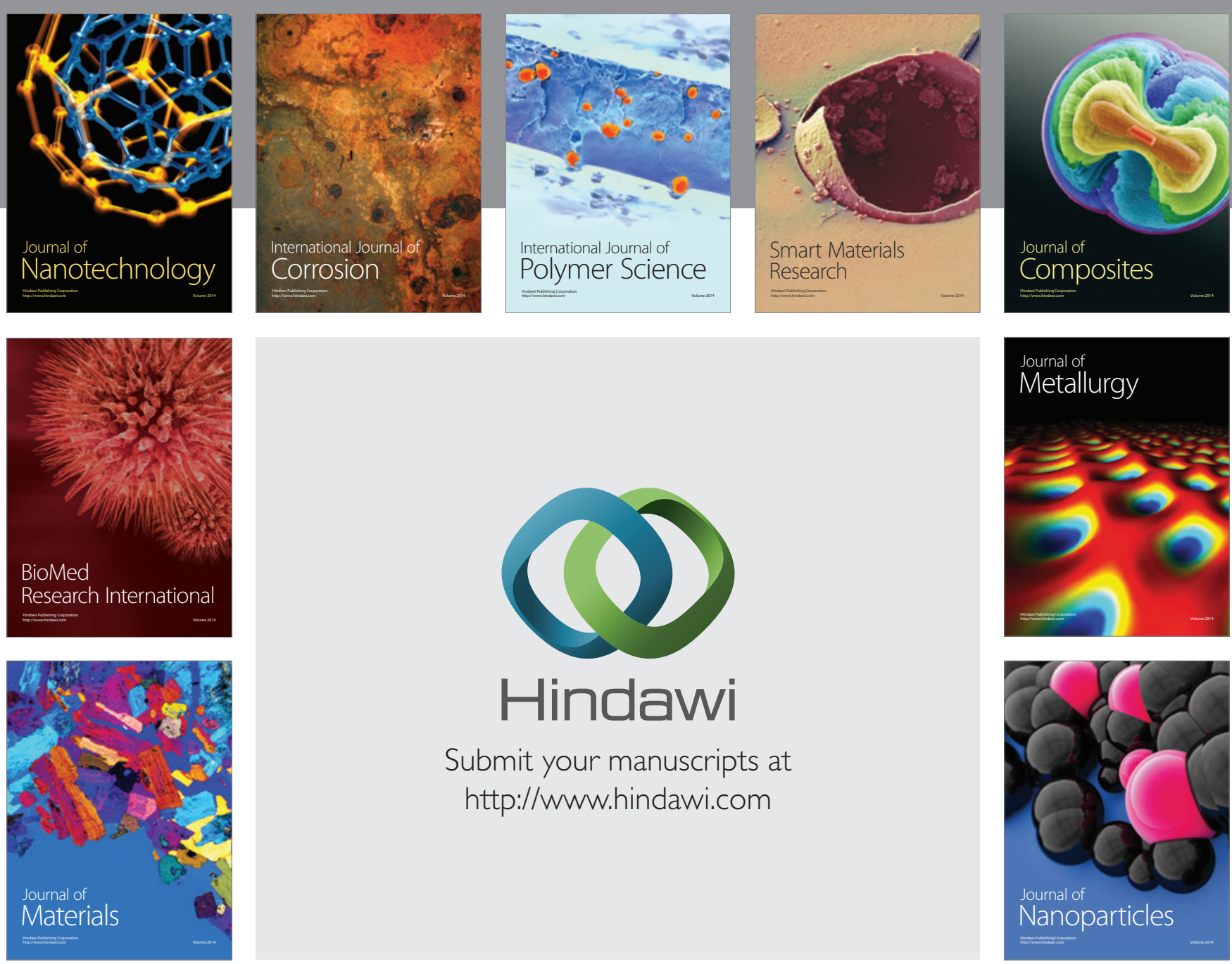

Submit your manuscripts at http://www.hindawi.com
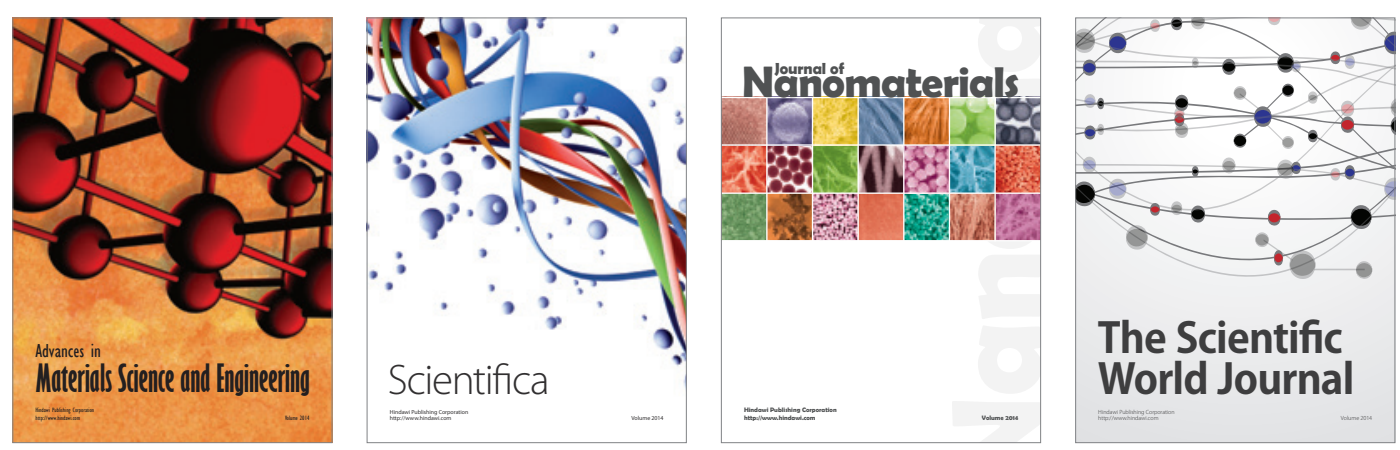

\section{The Scientific World Journal}
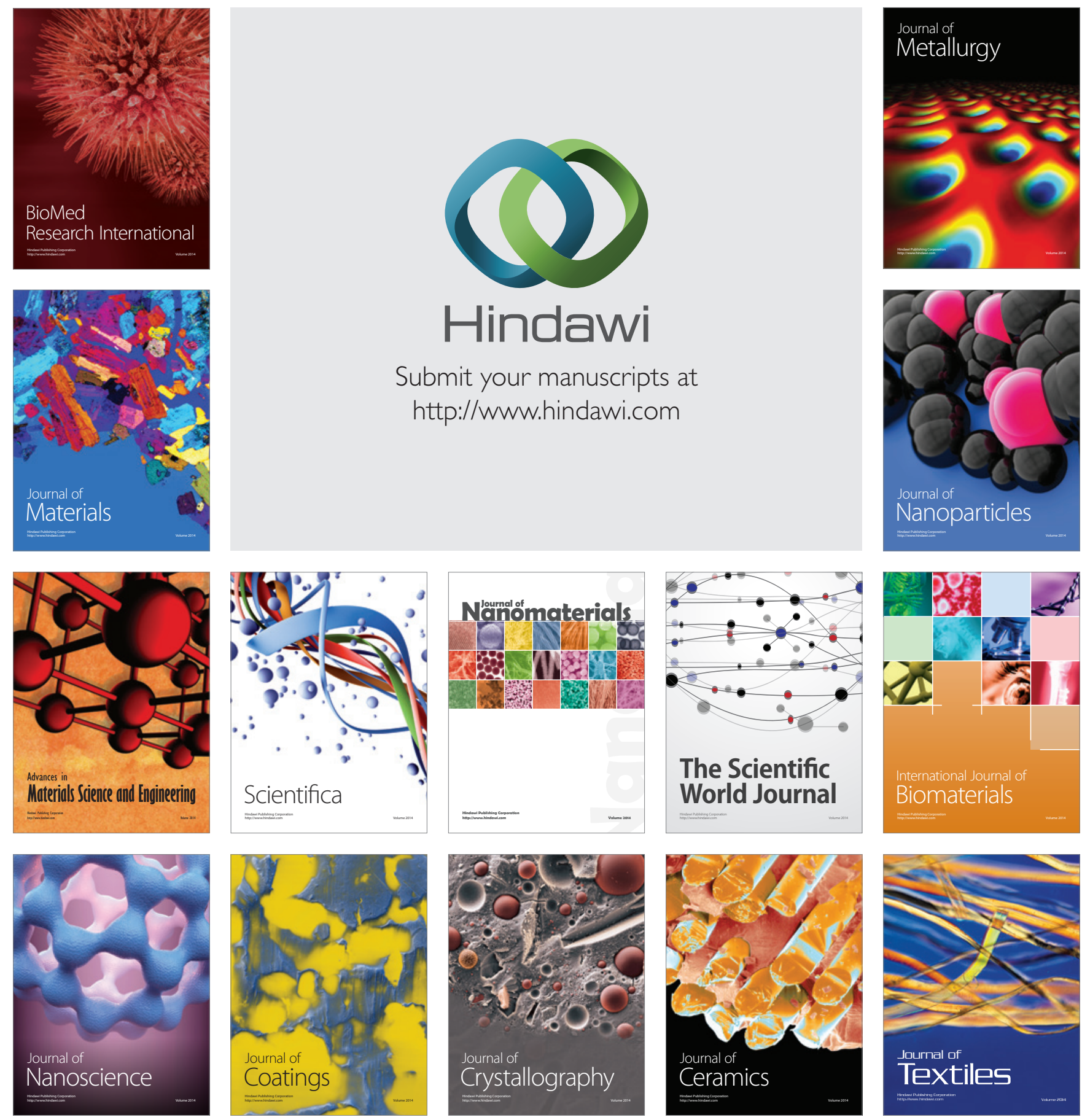